\title{
REGULARIZATION AND ASYMPTOTIC EXPANSION OF CERTAIN DISTRIBUTIONS DEFINED BY DIVERGENT SERIES
}

\author{
RICARDO ESTRADA \\ Formela de Matemática. Universidad de Conta Rica \\ Sall .losí. Costa Rioa \\ (Received May 4, 1994 and in revised form September 6, 1994)
}

\begin{abstract}
The regulariation of the distribution $\sum_{n=-x}^{x} \delta\left(x-\mu^{n}\right)$. which gives a regularized value to the divergent series $\sum_{n=-x}^{x} O\left(p^{\prime \prime}\right)$. is obtained in several spaces of test functuns. The asymptotic expansion as $z \rightarrow 0^{+}$of series of the type $\sum_{n=0}(\phi)\left(=p^{\prime \prime}\right)$ is also obtained
\end{abstract}

KEY WORDS AND PHRASES Generalized functions. asymptotic expansions

1992 AMS SUB.JECT CLASSIFICATION C(ODES. 41A60), 46F12

\section{INTRODUCTION}

Divergent series of the type

$$
\sum_{n=-\infty}^{\infty} \phi\left(p^{n}\right) . \quad \sum_{n=0}^{x} \phi\left(p^{n}\right) .
$$

where $p>1$ and where $\phi$ is a given function. as well as the aswmptotic development as $\varepsilon \rightarrow()^{\dagger}$ of the related series

$$
\sum_{n=-x}^{\infty} \phi\left(\varepsilon \mu^{n}\right) . \quad \sum_{n=0}^{x} \phi\left(\varepsilon \mu^{n}\right) .
$$

have been shown to be of importance in several areas, among which we could mention the counting algorithms used in database systems [8] and the use by Ramamujan of relaterl series, with $p=2$, in his approach to the problem of the distribution of prine numbers [10. lecture 2].

The ain of the present article is to provide a regularization of the generalized function

$$
y(x)=\sum_{n=-\infty}^{x} \delta\left(x-p^{n}\right) .
$$

where $\delta(x-\xi)$ is the Dirac delta function concentrated at the point $\xi$. so that the evaluation of $g(x)$ at the test function $\phi(x)$. $\langle y, \phi\rangle$, provides a regularized value to the generally divergent series

$$
\sum_{n=-x}^{\infty} \dot{\phi}\left(p^{r}\right)
$$

We give regularizations in the standard spaces of distributions $\mathcal{D}$ or $\mathcal{S}$ as well as in the' spaces $\mathcal{S}\left\{x^{\alpha_{n}}\right\}$ and $\mathcal{S}\left\{x^{\alpha^{\prime}} \ln x . x^{\alpha_{n}}\right\}$ introduced in $[6]$ to study distributional asymptotic expansions. The regularization is achieved by using the Hadamard finite part ideas. f'mployed in the regularization of distributions detined by divergent integrals $[5]$. 
Subsequently, we consider the asvmptotic developments (1.2) bv using the theory of asymptotic expansions of generalized functions. The close ties between generalized functions and asymptotic analysis have been studied by several anthors [1.3.4.6.7.12.14.15]. who have shown that generalized functions provide a very suitable framework for the asymptotic expansion of integrals and sories.

In the present study we obtain the expansion of $(1.2)$ as $\varepsilon \rightarrow 0^{+}$for sunoth functions with power or logarithmic behavior near the origin.

The plan of the article is as follows. In the serond section we briefly review some ideas from the theory of asymptotic expansions of distributions. The third sertion studies related expansions of convergent series. The regularization in $\mathcal{S}^{\prime}\left\{x^{\alpha_{n}}\right\}$ and in $\mathcal{S}^{\prime}\left\{x^{\alpha{ }^{\prime \prime}} \ln x, x^{\prime \prime \prime}\right\}$ is considered in the next two sections. respectively. where the asymptotic expansion is also given. The last section gives an ilustration of these results to the construction of counterexamples to some Hardy Littlewood type Tanberian theorems.

\section{PRELIMINARIES.}

In this section we provide a list of spaces of generalized functions needed in this paper. We also discuss the moment asymptotic expansion, a concept that plays a key role in our analysis.

The basic spaces of distributions that we are going to need are the spaces $\mathcal{D}^{\prime}(\mathbf{R}), \mathcal{S}^{\prime}(\mathbf{R})$ and $\mathcal{E}^{\prime}(\mathbf{R})$. The space of test functions $\mathcal{D}$ consists of the smooth functions with compact support. The space of test functions $\mathcal{S}$ consist of those smooth functions $\phi$ for which

$$
\phi^{(J)}(x)=O\left(|x|^{-n}\right), \quad \text { as } \quad|x| \rightarrow \infty .
$$

for each $J, n \geq 0$. The space $\mathcal{E}$ consists of all smooth functions with the topology of uniform convergence of all derivatives on compact sets. The dual spaces, $\mathcal{D}^{\prime} . \mathcal{S}^{\prime}$. and $\mathcal{E}^{\prime}$ are, respertively. the spaces of standard. tempered and compactly supported distributions. For det ails. see [11.13].

The moment asymptotic expansion in the space $\mathcal{E}$ takes the following form

THEOREM 1. Let $f \in \mathcal{E}^{\prime}$. Then

$$
f(\lambda x) \sim \sum_{n=0}^{\infty} \frac{(-1)^{n} \mu_{n} \delta^{(n)}(x)}{n ! \lambda^{n+1}}, \quad \text { as } \quad \lambda \rightarrow \infty
$$

where

$$
\mu_{n}=\left\langle f(x), x^{n}\right\rangle
$$

are the inoments.

The moment asymptotic expansion also hold in other spaces such as $\mathcal{O}_{(:}^{\prime}, \mathcal{O}_{M}^{\prime}$. $\mathcal{K}^{\prime}$ or $\mathcal{P}^{\prime}$ of distributions of "rapid decay at infinity". but it does not hold in $\mathcal{S}^{\prime}$ nor $\mathcal{D}^{\prime}[6.7]$. At present we shall have use for the result in $\mathcal{E}^{\prime}$ only.

Another class of spaces of generalized functions, very suitable for the study of asymptotic expansions. is the following. Let $\left\{\phi_{n}(x)\right\}$ be a sequence of smooth functions defined in $(0 . \infty)$ 


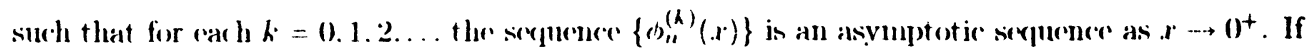
$\mathcal{A}$ is any space of test functions. such as $\mathcal{E}$. $\mathcal{D}$. $\mathcal{S}$ or other. then the space $\mathcal{A}\{0$, $(x)\}$ consists of those smooth functions $\psi(x)$ defined in $(0 . x)$ which show the behavior of the trpe of the spare $\mathcal{A}$ as $r \rightarrow x$ while for small $x$ admit the asvinptotic expansion for $k=0.1$. 2. .

$$
a^{(k)}(x) \sim a_{1} b_{1}^{(k)}(x)+a_{2} b_{2}^{(h)}(x)+a_{3} o_{3}^{(k)}(x)+\cdots \quad \text { as } \quad r \cdots 0^{+} .
$$

where $a_{1} \cdot a_{2} \cdot a_{3} \ldots$ are cert ain constants.

The functionals $\lambda_{n} \in \mathcal{A}^{\prime}\left\{o_{n}\right\}$ are given as

$$
\left\langle\lambda_{n}(x) \cdot u \cdot(x)\right\rangle=a_{n} .
$$

The generalized moment asymptotic expansion in the space $\mathcal{E}^{\prime}\left\{x^{\prime \prime \prime}\right\}$. where $\left\{x_{n}\right\}$ is a serpuence with fic'on, $/ x$. takes the form

$$
f(\lambda . r) \sim \sum_{n=1}^{x} \frac{\mu\left(\alpha_{n}\right) \lambda_{n}(r)}{\lambda^{\prime n}+1} . \quad \text { as } \quad \lambda \rightarrow x .
$$

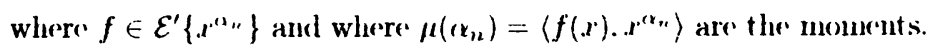

A moment expansion also holds in the space $\mathcal{E}^{\prime}\left\{x^{\alpha_{n}} \ln , x_{,}, r^{\alpha_{n}}\right\}$. If we use the notation $\delta_{n}(x)$ and $\delta_{u}^{\prime}(x)$ for the functionals defined as

$$
\begin{aligned}
& \left\langle\delta_{n}(x) \cdot \psi(x)\right\rangle=a_{n} . \\
& \left\langle h_{11}^{\prime}(x) \cdot \psi(x)\right\rangle=a_{n}^{\prime} .
\end{aligned}
$$

for a function $\psi^{\prime} \in \mathcal{E}\left\{x^{\alpha_{n}} \ln . x . x^{\alpha_{n}}\right\}$ with expansion

$$
\psi(x) \sim \sum_{n=1}^{\infty}\left(a_{n}^{\prime} \ln x+a_{n}\right) x^{\alpha_{n}} . \quad \text { as } \quad x \rightarrow 0^{+} .
$$

then the moment asymptotic expansion takes the form

$$
f(\lambda x) \sim \sum_{n=1}^{\infty} \frac{-\mu^{\prime}\left(\alpha_{n}\right) \delta_{n}^{\prime}(x) \ln \lambda+\left[\mu^{\prime}\left(\alpha_{n}\right) \delta_{n}^{\prime}(x)+\mu\left(\alpha_{n}\right) \delta_{n}(x)\right]}{\lambda^{\alpha_{n}+1}}
$$

as $\lambda \rightarrow \infty$, where the $\mu^{\prime}\left(\alpha_{n}\right)=\left\langle f(x) . x^{\alpha_{n}} \ln x\right\rangle$ are the logarithmic moments.

\section{EXPANSION IN THE SPACE $\mathcal{S}$.}

In this section we consider the asymptotic expansion as $\varepsilon \rightarrow 0^{+}$of series of the typo

$$
\sum_{n=0}^{\infty} p^{n} \phi\left(\varepsilon p^{n}\right)
$$

when the function $\phi$ belongs to the space $\mathcal{S}$. Here $p$ is a constant. $p>1$.

Notice that the values of $\phi(x)$ for negative $x$ are irrelevant. What matters is that $\phi$ is smooth near $r=0$ and thus the one-sided Taylor expansion 


$$
\phi(x) \sim c(0)+x c^{\prime}(x)+\frac{x^{2}}{2 ! !}()^{\prime \prime}(x)+\cdots \quad \text { as } \quad r \rightarrow 0^{+}
$$

bolds.

In order to study (3.1) we introduce the there generalized functions $f(x)$. $f_{+}(x)$ and $f-(x)$. defined as follows:

$$
\begin{aligned}
& f(x)=\sum_{n=-x}^{x} p^{\prime \prime} \lambda\left(x-p^{n}\right) . \\
& f_{+}(x)=\sum_{n=1}^{x} p^{\prime \prime} \lambda\left(x-p^{\prime \prime}\right) . \\
& f_{-}(x)=\sum_{n=1}^{x} p^{-\prime \prime} \lambda\left(x-p^{-n}\right) .
\end{aligned}
$$

Ohserve that $f$. $f_{+}$and $f_{-}$are positive Radon measures and that

$$
f(x)=f_{+}(x)+f_{-}(x)
$$

Notice also that (3.1) can be written as

$$
\sum_{n=0}^{x} p^{\prime \prime} \phi\left(\varepsilon p^{\prime \prime}\right)=\varepsilon^{-1}\left\langle f_{+}(x) \cdot(\dot{\varphi})(\varepsilon \cdot x)\right\rangle=\left\langle f_{+}(\lambda \cdot x) \cdot \phi(x)\right\rangle .
$$

where $\lambda=\varepsilon^{-1}$.

Let us start with the dist ribution $f\left(x^{\prime}\right)$. Clearly. $f \in \mathcal{S}^{\prime}$. Moreever.

$$
f(p \cdot x)=f(x)
$$

as follows directlv from (3.3a). Thus

$$
f(x)=F(\ln x) .
$$

where $F$ is perisdic of period lu $p$. The mean of $F$. its average over an interval of lengtin $\ln p$, is

$$
\frac{1}{\ln p} \int_{\alpha}^{\alpha+\ln p} F(u) d u=\frac{1}{\ln p} \int_{e^{\alpha}}^{p e^{\alpha}} f(x) \frac{d x}{x}=\frac{1}{\ln p} .
$$

Therefore we can write

$$
f(x)=\frac{H(x)}{\ln p}+g(x)
$$

where $H(x)$ is the Heaviside function and where $g$ is a periodic function of $\ln x$ of period $\ln p$ and zero mean. It follows that if $\phi \in \mathcal{S}$ then

$$
\sum_{n=-x}^{\infty} p^{n} \phi\left(\varepsilon p^{n}\right)=\frac{1}{\varepsilon \ln p} \int_{0}^{\infty} \phi(x) d x+\frac{\psi(\varepsilon)}{\varepsilon}
$$

where the oscillat ory component $\psi(\varepsilon)=\psi(\phi: \varepsilon)$. given by

$$
\varepsilon: \varepsilon)=\varepsilon(g(x) \cdot \phi(\varepsilon x)\rangle
$$

is a periodic function of $\ln \varepsilon$ of period lin $p$ and zero mean. 
Next. let us comsider the generalized function $f_{--}(x)$. It has compact support and thus $f_{-}\left(\lambda_{x}\right)$ adumits the moment asimptotic expansion as $\lambda \rightarrow x$.

Thr moments are

$$
\mu_{k}=\left\langle f_{-}(x), x^{k}\right\rangle=\sum_{n=1}^{j} p^{-" n} p^{n k}=\frac{1}{p^{k+1}-1}
$$

honer

$$
f_{-}(\lambda . r) \sim \sum_{k=0}^{x} \frac{(\cdots 1)^{k} \lambda^{(k)}(r)}{k !\left(p^{k+1}-1\right) \lambda^{k+1}} . \quad \text { ass } \quad \lambda \cdots, x .
$$

Combining (3.4). (3.9) and (3.13) we obtain the development of $f_{+}(\lambda x)$ as

$$
f_{+}(\lambda x) \sim \frac{H(x)}{\ln p}+g(\lambda x)-\sum_{k=1}^{x} \frac{(-1)^{k} \lambda^{(\alpha)}(x)}{k !\left(p^{k+1}-1\right) \lambda^{k+1}} \text {. as } \lambda \rightarrow x \text {. }
$$

Evaluating (3.14) at $\phi \in \mathcal{S}$ and setting $\lambda=\varepsilon^{-1}$ viclds the expansion

$$
\sum_{n=11}^{2} p^{n} \phi\left(\varepsilon p^{n}\right) \sim \frac{1}{\varepsilon \ln p} \int_{0}^{x} \phi(x) d x+\frac{\varepsilon(\varepsilon)}{\varepsilon} \cdot \sum_{k=0}^{x} \frac{\phi^{(h)}(0) \varepsilon^{h}}{k !\left(p^{h+1}-1\right)} .
$$

\section{REGULARIZATION IN THE SI'ACE $\mathcal{S}^{\prime}\left\{x^{\prime \prime \prime}\right\}$.}

Notice that (3.15) does not give the asymptotic development of the screses $\sum_{n=0}^{x} \phi\left(p^{n}\right)$. for $\phi \in \mathcal{S}$, unless $\phi(0)=0$. Wi now attend to this problem. in the more general fratuework of the spaces $\mathcal{S}\left\{x^{* n}\right\}$. Inderel. the development of $\sum_{n=0}^{x} p^{\prime \prime} 6\left(c p^{\prime \prime}\right)$ for $\psi ; \in \mathcal{S}\left\{r^{-1} .1, \ldots \ldots\right\}$ wonld give the expansion of $\sum_{n=0}^{\infty} \phi\left(\varepsilon p^{n}\right)$ for $\phi \in S$ by setting $\psi(x)=x^{-1} \phi(x)$

Even though the seriess $\sum_{n=0}^{x} p^{n} \phi\left(p^{n}\right)$ converges for $\phi \in \mathcal{S}\left\{x^{\alpha_{n}}\right\}$ for any serjuence $\left\{x_{n}\right\}$ with Rea $\alpha_{n} x$. the method of the previous sertion requires the consideration of the two generally divergent series

$$
\begin{aligned}
\langle f(x) . \phi(x)\rangle & =\sum_{n=-\infty}^{x} p^{n} \phi\left(p^{n}\right), \\
\left\langle f_{-}(x) . \phi(x)\right\rangle & =\sum_{n=1}^{\infty} p^{-n} \phi\left(p^{-n}\right) .
\end{aligned}
$$

Therefore, we study the regularization of $f(x)$ and $f_{-}(x)$ in the spaces $\mathcal{S}\left\{x^{\prime \prime \prime}\right\}$. Naturally the two regularization problems are equivalent in view of (3.5).

Let $\phi \in \mathcal{S}\left\{x^{x_{n}}\right\}$. with expansion

$$
\phi(x) \sim a_{1} \cdot x^{\alpha_{1}}+a_{2} y^{\alpha_{2}}+a_{3.3} x^{\alpha_{3}}+\cdots \quad \text { ass } r \rightarrow 0^{+} .
$$

Suppose $\alpha_{m}=-1$. Then the series giving the value of $\left\langle f_{-}(x) . \phi(x)-\sum_{j=1}^{m} a, x^{n j}\right\rangle$ converges. Thus it is chough to give the finite part of the sum $\sum_{n=1}^{x} p^{-\prime} \phi\left(y^{-n}\right)$ if $\phi(x)=x^{a}, \alpha \leq-1$. If $\alpha=-1$ we have 


$$
\sum_{n=1}^{N} p^{-n} p^{-n n}=\frac{p^{-(1+n)}-p^{N(1+n)}}{1-p^{-1+n}}
$$

thus the partial sums consist of two parts. namely. the intinite part $\frac{p^{-N(1+c)}}{1-p^{-(1+0)}}$. and the finite part

$$
\left\langle f_{-}(r) . r^{\prime \prime}\right\rangle=\mathrm{FP} \sum_{n=1}^{\infty} p^{-n(1+r)}=\frac{1}{p^{1+r}-1} .
$$

When $\alpha=-1$ wo have

$$
\left\langle f_{-}(x) . l^{-1}\right\rangle=\mathrm{F}_{\mathrm{P}} \sum_{n=1}^{\mathrm{x}} 1=\mathrm{F}_{\mathrm{P}} \lim _{N \rightarrow \chi} N=0 .
$$

Therefore, the regularization of $f_{--}(x)$ is given by

$$
\left\langle f_{-}(x) . \phi(x)\right\rangle=\sum_{j=1}^{m-1} \frac{n_{j}}{p^{1+(x,-1}}+\sum_{n=1}^{x} p^{-n}\left(c\left(p^{-n}\right)-\sum_{j=1}^{m} n t_{j}^{-m x_{j}}\right) .
$$

if $\phi \in \mathcal{S}\left\{r^{\alpha{ }}\right\}$ has the expansion (4.2).

The regularization of $f(x)$ is given by the formula $f=f_{+}+f$. since $f_{+}$is a well-definert element of $\mathcal{S}^{\prime}\left\{x^{\alpha_{n}}\right\}$. Observe that if $o \in \mathcal{S}\left\{x^{n_{n}}\right\}$ has the development (4.2) then

$$
\begin{aligned}
& \langle f(p x) \cdot \phi(x)\rangle=\frac{1}{p}\langle f(x) \cdot \phi(x / p)\rangle \\
& =\frac{1}{p}\left[\sum_{j=1}^{m-1} \frac{a_{j} p^{(x)}}{p^{1+\alpha_{j}}-1}+\sum_{n=1}^{x} p^{-n}\left(\dot{\phi}\left(p^{-n-1}\right)-\sum_{j=1}^{m} a_{j} p^{-(n+1)\left(r_{j}\right.}\right)+\sum_{n=0}^{x} p^{n} \phi\left(p^{n}\right)\right] \\
& =\sum_{j=1}^{m-1} \frac{a_{j}}{p^{1+\omega_{j}}\left(p^{1+n_{j}}-1\right)}+\sum_{n=1}^{\infty} p^{-n}\left[\phi\left(p^{-n}\right)-\sum_{j=1}^{m} a, p^{-n \alpha_{j}}\right]+\sum_{n=0}^{\infty} p^{n} \phi\left(p^{n}\right)+\sum_{j=1}^{m} a_{j} p^{-\omega_{j}-1} \\
& =\langle f(x) . \phi(x)\rangle \text {. }
\end{aligned}
$$

so that $f(x)$ is still a periodic fimction of $\ln x$ in the space $\mathcal{S}\left\{x^{\alpha_{n}}\right\}$. Hence we can write. as before.

$$
f(x)=\frac{H(x)}{\ln p}+g(x)
$$

where the oscillatory generalized function $g(x)$ has zero mean.

Notice. however, that the formula $H(\lambda x)=H(x)$ ceases to hold in the space $\mathcal{S}\left\{x^{\prime \prime \prime}\right\}$.

Indeed, if $\phi \in \mathcal{S}\left\{x^{\alpha_{n}}\right\}$, the integral $\int_{0}^{\infty} \phi(x) d x$ is generally divergent and thus it is necessary to consider its finite part $\mathrm{Fp}_{\mathrm{p}} \int_{0}^{\infty} \phi(x) d x$. But if $\alpha_{m}=-1$, then

$$
\mathrm{Fp} \int_{0}^{\infty} \phi(\varepsilon x) d x=\frac{1}{\varepsilon}\left[\mathrm{Fp} \int_{0}^{\infty} \phi(x) d x-a_{m} \ln \varepsilon\right]
$$

so that

$$
H\left(\lambda . x^{\prime}\right)=H(x)+\frac{\ln \lambda}{\lambda} r_{m}(x) .
$$

where $\delta_{m}(x)$ is the functional defined in (2.5). so that $\left\langle\lambda_{m} . \phi\right\rangle=a_{m}$.

The moment asymptotic expansion of $f_{-}(\lambda x)$ in the space $\mathcal{S}\left\{x^{\prime \prime \prime}\right\}$ takes the form

$$
f .(\lambda . x) \sim \sum_{j=1}^{m-1} \frac{\partial_{j}(r)}{\left(p^{1+r_{j}}-1\right) \lambda^{\left(x_{j}\right.}}+\sum_{j=m+1}^{x} \frac{\lambda_{j}(x)}{\left(p^{1+r_{j}}-1\right) \lambda^{\alpha_{j}}} . \quad \text { as } \lambda \rightarrow x \text {. }
$$


If we now use (4.6). (4.8) and (4.9). we obtain the expansion of $f_{+}(\lambda x)$ as

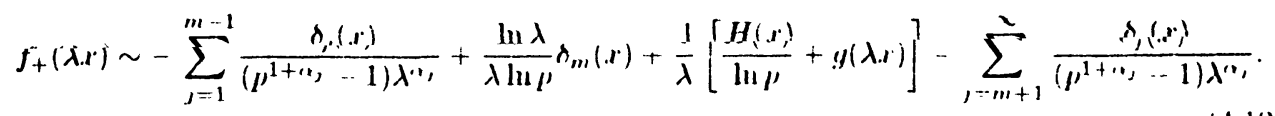

Sort $\varepsilon=\lambda^{1}$. Then "waluation at o $\in \mathcal{S}\left\{r^{\prime \prime \prime}\right\}$ yiolds

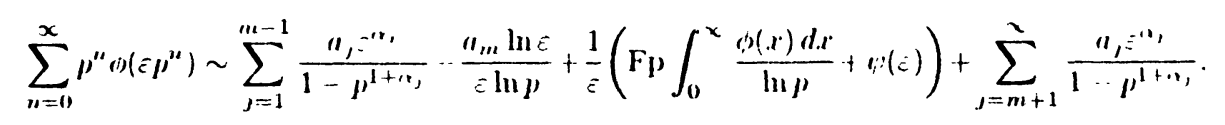

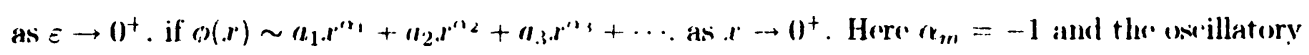
component $\theta(\varepsilon)=(\cdot)(\phi: \varepsilon)$ is a periodic function of ln $\varepsilon$ of poriod hi $p$ and wero mean.

Let us now replace $\phi(x)$ by $g(x) / x$ in formula $(4.11)$. Since $\phi(x) / x$ has the expansion $a_{1} \cdot x^{a_{1}-1}+a_{2} \cdot x^{a_{2}-1}+a_{3} \cdot x^{n_{3}-1}+\cdots$ as $x \rightarrow 0^{+}$, we obtain the formula

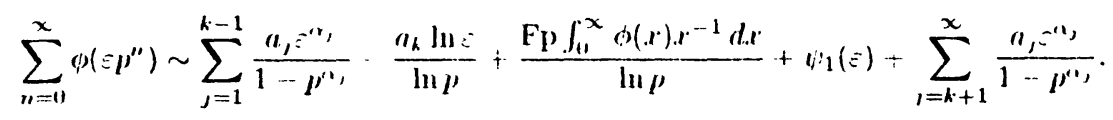

where $\alpha_{k}=0$ and where $\psi_{1}(\varepsilon)$ is a periodic function of $\ln \varepsilon$ of period $\ln p$ and zero ment

In particular. if $\varphi \in \mathcal{S}$ formula (4.12) takes the form

$$
\sum_{n=0}^{\infty} \phi\left(\varepsilon p^{n}\right) \sim \frac{-\phi(0) \ln \varepsilon}{\ln p}+\frac{\mathrm{F}_{p} \int_{0}^{\infty} \phi(x) x^{-1} d x}{\ln p}+\psi_{1}(\varepsilon)+\sum_{n=1}^{\infty} \frac{i^{(j)}(n)(0) \varepsilon^{n}}{n !\left(1-p^{n}\right)} .
$$

5. REGULARIZATION IN $\mathcal{S}^{\prime}\left\{x^{\alpha_{*}} \ln \cdot x^{\prime}, x^{\left(x_{*}\right.}\right\}$.

The same regularization procerlure can be applied to the space $\mathcal{S}^{\prime}\left\{x^{\prime \prime}, \ln x . . x^{\alpha{ }^{\prime \prime}}\right\}$. Indeerl. we use the finite part values

$$
\begin{aligned}
\left\langle f_{-}(x) \cdot x^{-1} \ln x\right\rangle & =-\ln p \mathrm{~F} p \sum_{n=1}^{\infty} n=0 . \\
\left\langle f_{-}(x) \cdot x^{\alpha} \ln x\right\rangle & =-\ln p \sum_{n=1}^{\infty} n p^{-n(\alpha+1)}=\frac{-p^{1+\alpha} \ln p}{\left(p^{1+\alpha}-1\right)^{2}} . \quad a<\cdots 1 .
\end{aligned}
$$

to obtain the regularization of $f_{-}(x)$ and. consecquently. of $f(x)$. The gencrulized function $f(x)$ remains a periodic function of $\ln x$, of period $\ln p$.

Formula (4.7) becomes

$$
\mathrm{Fp} \int_{0}^{\infty} \phi(\varepsilon x) d x=\frac{1}{\varepsilon}\left[\mathrm{Fp} \int_{0}^{x} \phi(x) d x-a_{m} \ln \varepsilon-a_{m}^{\prime} \frac{(\ln \varepsilon)^{2}}{2}\right] .
$$

if $\phi(x) \sim \sum_{j=1}^{x}\left(a_{j}^{\prime} \ln x+a_{j}\right): x^{\alpha_{j}}$. as $x \rightarrow 0^{+}$and $\alpha_{m}=-1$.

The asymptotic formula then takes the form

$$
\begin{aligned}
& \sum_{n=0}^{\infty} \phi\left(\varepsilon \rho^{n}\right) \sim \sum_{j=1}^{k-1}\left[\frac{a_{j}^{\prime} \ln \varepsilon+a_{1}}{1-p^{\alpha_{j}}}+\frac{a_{j}^{\prime} p^{\alpha_{j}} \ln p}{\left(1-p^{\prime \prime}\right)^{2}}\right] \varepsilon^{\prime \prime} \\
& +\frac{\mathrm{Fp}_{\mathrm{p}} \int_{0}^{\infty} \phi(x) r^{-1} d r}{\ln p^{\prime}}-\frac{a_{k} \cdot \ln \varepsilon}{\ln p}-\frac{a_{k}^{\prime}(\ln \varepsilon)^{2}}{2 \ln p}+4:(\varepsilon)
\end{aligned}
$$

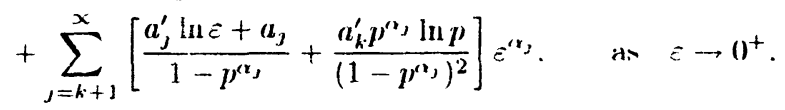


whrere $\alpha_{k}=0$.

\section{AN ILLUSTRATION.}

There are some Tauberian theorems, which go back to Hardy and Littlewool [9]. for the differentiation of asymptotic approximations which are valid under yppropiate monotonicity conditions [2]. For instance. if

$$
G(x) \sim p^{\lambda r} . \quad \text { as } \quad x \rightarrow \infty .
$$

where $\lambda \neq 0$, and if $G^{\prime}$ is increasing. then

$$
G^{\prime}(x) \sim e^{\lambda x}
$$

The corresponding Tauberian theorems for comparison with a power function also hold, namely, if

$$
F(x) \sim(x-a)^{a} . \quad \text { as } \quad x \rightarrow a^{+}
$$

and if $F^{\prime}$ is increasing and $\alpha>1$ or $\alpha<0$ or $F^{\prime}$ is decreasing and $0<\alpha<1$, then

$$
F^{\prime}(x) \sim \alpha(x-a)^{\alpha-1}, \quad \text { as } \quad x \rightarrow a^{+} .
$$

Therefore, it is surprising that the results for comparison with a logarithuic function are false [2]. A simple counterexample can be constructed by using the asymptotic upproximations of the previous sections. Indeed, let $p>1$ and set

$$
f(x)=\sum_{n=0}^{\infty} x^{p^{n}}, \quad 0<x<1
$$

The behavior of $f(x)$ as $x \rightarrow 1^{-}$can be found from (4.13) by taking $x=e^{-\varepsilon}, \varepsilon=\ln (1 / x)$ and $\phi(x)=e^{-x}$. The result is

$$
f(x) \sim \frac{-\ln \ln (1 / x)}{\ln p}-\gamma+\omega(\ln \ln (1 / x))+\sum_{n=1}^{\infty} \frac{(\ln x)^{n}}{n !\left(1-p^{n}\right)}, \quad \text { as } \quad x \rightarrow 1^{-},
$$

where we have used the value $\gamma=\int_{0}^{\infty} \frac{e^{-x}}{x} d x$, Euler's constant, and where $\omega$ is a periodic function of period $\ln p$ and zero mean.

To the first order, (6.6) takes the form

$$
f(x) \sim \frac{\ln \left(\frac{1}{1-x}\right)}{\ln p}, \quad \text { as } \quad x \rightarrow 1^{-}
$$

But $f^{\prime}(x)$, though increasing, is not asymptotically equivalent to $\frac{1}{(1-x) \ln p}=\frac{d}{d x}\left[\frac{\ln \left(\frac{1}{1-x}\right)}{\ln p}\right]$. Actually, using (4.11) we find the approximation

$$
\begin{aligned}
f^{\prime}(x) & =\sum_{n=0}^{\infty} p^{n} x^{p^{n}-1}=\frac{1}{x} \sum_{n=0}^{\infty} p^{n} x^{p^{n}} \\
& \sim \frac{1}{x}\left[\frac{1}{\ln p \ln (1 / x)}+\omega_{1}(\ln \ln (1 / x))+\sum_{n=0}^{\infty} \frac{(\ln x)^{n}}{n !\left(1-p^{n+1}\right)}\right], \quad \text { as } \quad x \rightarrow 1^{-} .
\end{aligned}
$$


To the first order. then.

$$
f^{\prime}(x) \sim \frac{1}{1 \cdots x}\left[\frac{1}{\ln p}+u^{\prime}\left(\ln \ln \frac{1}{r}\right)\right] .
$$

so that $f^{\prime}(x)(1-x) \ln p$ does not tend to 1 . but rat her it oscillates about it since $\omega_{1}$ is a periodic: function with \%ero medn.

\section{REFERENCES}

1. BRÜNING. J. On the asvmptotic expansion of some integrals. Arch. Math. $\underline{58}$ (1984), 253 259.

2. DURÁN. A. L. El recílproco de la regla de L'Hòpital. Rev. Cienc. Tern. (C.R.) 16 (1992). $111 \cdot 119$.

3. ESTRADA, R. The asymptotic expansion of certain series considered by Ramanujan, Appl. Anal. 43 (1992). $191-228$.

4. ESTRADA, R., GRACIA-BONDIA. J. M. and VARILLY. J. C.. On asymptotic expansions of twisted products, J. Math. Phys. 30 (1989). 27892796.

5. ESTRADA, R. and KANWAL, R. P. Regularization, pseudofunction and Hardamard finite part, J. Math. Anal. Appls. 141 (1989), 195-207.

6. ESTRADA, R. and KANWAL, R. P. A distributional theory for asymptotic expansions. Proc. Roy. Soc. London A 428 (1990). 399-430.

7. ESTRADA, R. and KANWAL, R. P. The asymptotic expansion of certain multidimensional generalized functions, J. Math. Anal. Appl. 163 (1992), 264-283.

8. FLAJOLET, P. and MARTIN, G. N. Probabilistic counting algorithms for database applications, J. Comp. Syst. Sci. $\underline{31}$ (1985), 182209.

9. HARDY, G. H. Divergent Series, Clarendon Press, Oxford, 1949.

10. HARDY, G. H. Ramanujan: Twelve lectures on Subjects suggested by his Life and Work, Chelsea, New York, 1978.

11. KANWAL, R. P. Generalized Functions: Theory and Terhnique. Academic Press, New York, 1983.

12. PILIPOVIC, S. Quasiasymptotic expansion and Laplace transform, Appl. Anal. $\underline{35}$ (1990), 247-261.

13. SCHWARTZ. L. Thèorie des Distributions, Hermann. Paris. 1966.

14. VLADIMIROV, V. S.. DROZHINOV, Y. N. and ZAVALOV, B. I., Multidimensional Tauberian Theorems for Generalized Functions, Nauka, Moskow, 1986.

15. WONG. R. Asymptotic Approximation of Integrals, Academic Press, New York. 1989. 


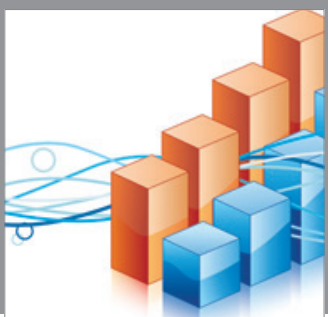

Advances in

Operations Research

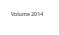

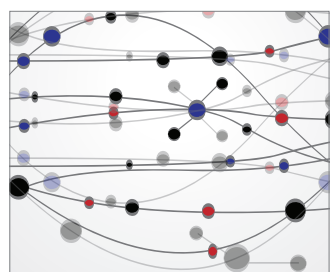

\section{The Scientific} World Journal
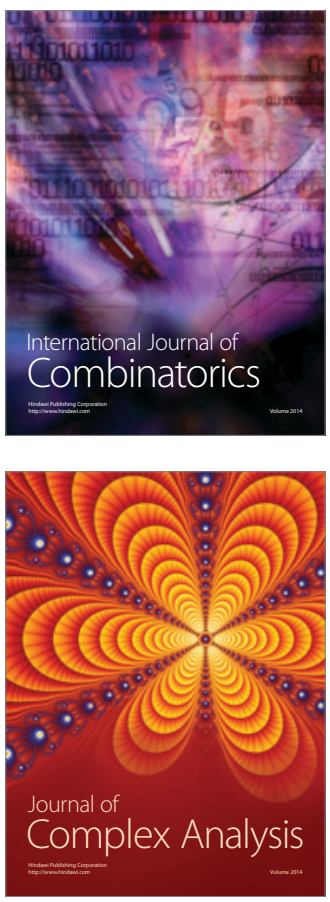

International Journal of

Mathematics and

Mathematical

Sciences
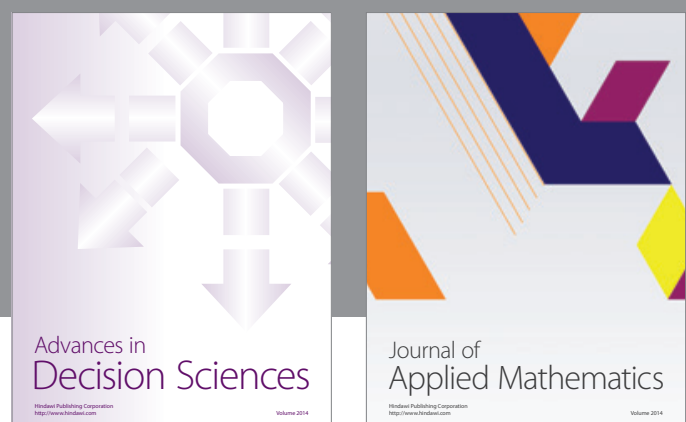

Journal of

Applied Mathematics
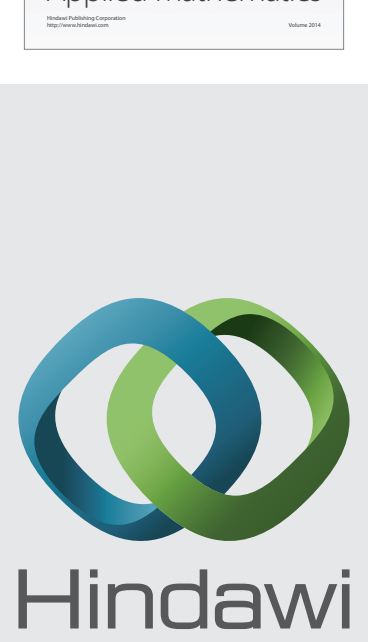

Submit your manuscripts at http://www.hindawi.com
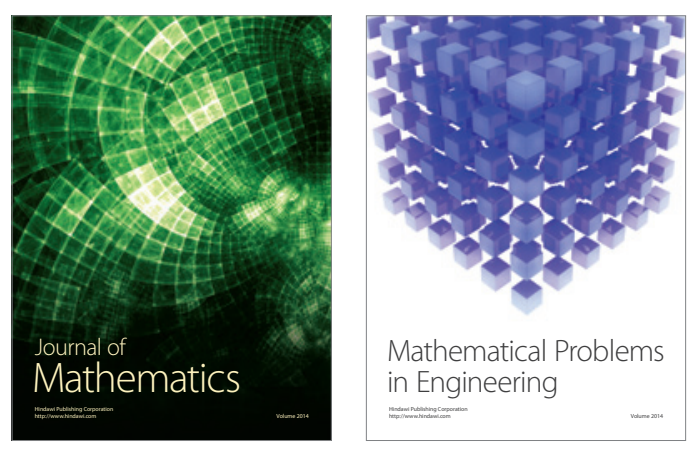

Mathematical Problems in Engineering
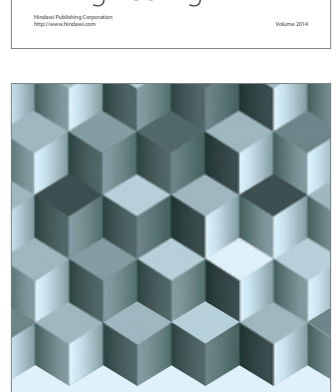

Journal of

Function Spaces


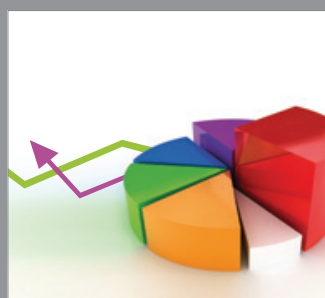

ournal of

Probability and Statistics

Promensencen
